\title{
Use of Dantrolene and Ativan to Treat Overlapping Diagnosis NMS Versus Malignant Catatonia Case Report
}

Dynela Garcia-Baran ( $\sim$ dynela.I.garciabaran.mil@mail.mil)

NMCP: Naval Medical Center Portsmouth https://orcid.org/0000-0001-8481-7074

\section{Sam Collier}

NMCP: Naval Medical Center Portsmouth

\section{Alejandro Ortiz}

University of Maryland School of Medicine

\section{Research Article}

Keywords: Catatonia, catatonia rating scale, neuroleptic malignant syndrome, malignant catatonia

Posted Date: November 9th, 2021

DOI: https://doi.org/10.21203/rs.3.rs-654688/v1

License: (9) (1) This work is licensed under a Creative Commons Attribution 4.0 International License. Read Full License 


\section{Abstract}

We present a case report of a patient who developed symptoms resembling malignant catatonia and neuroleptic malignant syndrome. Suspicion of neuroleptic malignant syndrome arose after treatment over his course of hospital stay with three different secondgeneration antipsychotics for a first-time bipolar type I manic episode. After a hospital stay of 5 days, the patient developed symptoms that could be interpreted as malignant catatonia or neuroleptic malignant syndrome. Administration of antipsychotics was immediately ceased, and the patient was transferred to the ICU where he was treated with dantrolene and higher dosages of Ativan. The patient improved after simultaneous intervention for both possible diagnoses. After approximately one month, quetiapine, one of the second generation antipsychotics previously prescribed, was restarted with good results and no reoccurrence of NMS or malignant catatonia. This case illustrates the potential dilemma faced when differentiation between the two obscure diagnoses is necessary. Diagnosis is typically established through clinical observation and monitoring of symptom evolution after the administration of neuroleptics. The treatment algorithms for each diagnosis vary as can the respective outcomes. Our case also highlights the dearth of research available on distinguishing neuropathologic psychiatric disorders from pathophysiologic psychomotor syndromes. It also focuses on the need for sound diagnostic scoring scales that will clarify the diagnostic picture as well as treatment guidelines to ensure best outcomes.

\section{Background:}

\section{Definition}

Catatonia is a marked deterioration in psychomotor function and depressed responsiveness. It can occur in the setting of a variety of primary medical or mental health conditions. It involves characteristic motor, cognitive, affective, and autonomic disturbances, and can include stupor, mutism, negativism and posturing (Spiegel et al., 2019). Catatonia per the DSM-5 is characterized by marked psychomotor disturbances that range from decreased motor activity to engagement in excessive peculiar motor activity. Thus, a subject can present with extreme unresponsiveness to heightened agitation (APA-DSM-5, 2013). Ativan, often prescribed for catatonia, has an effective treatment response of over $80 \%$.

Neuroleptic Malignant Syndrome (NMS) is generally considered to be an entity distinct from catatonia. Due to a significant clinical overlap of symptoms, however, they are often considered concurrent when a patient presents with the typical symptoms. Catatonia presents $73-78 \%$ of the time with rigor and mutism while NMS is more likely to present with fever and rigor in $85-87 \%$ of cases (Lang, Silke \& Thomas, 2014). Both conditions can cause significant harm. Catatonia can be associated with malnutrition, exhaustion, hyperpyrexia and self-inflicted injury or failure to care for self over time, whereas NMS is a more abrupt surge of symptoms that can cause more immediate mortality after neuroleptics are administered (Kumar et al., 2016).

Typically, catatonia is diagnosed in an inpatient setting with about $65 \%$ of cases found in patients with depression or bipolar disorders. Those with bipolar disorder can suffer with a physiologic and psychological burden that leads to a severe increase in morbidity. Some of these issues develop into manic delirium, catatonia, and NMS rather than malignant catatonia (Singh \& Akinyemi, 2005).

Evidence from database studies DSM-5 (2013, p. 710) suggests an incidence rate for NMS of $0.01-0.02 \%$ in patients treated with antipsychotics. Many of these patients, whether due to administration of antipsychotics or progression of their disease, can develop an increase in morbidity, including delirium, malignant catatonia, NMS, or catatonia. NMS is typically induced by exposure to dopamine antagonists and is clinically significant for hyperthermia, muscle rigidity, mental status alteration, autonomic instability and elevated creatine kinase levels (Rooijen et al., 2017). Additionally, it is advised that the clinician should have a high degree of suspicion for either of these disease processes due to the lethality associated with their progression (Singh \& Akinyemi, 2005). A well-known scale used for detection of catatonia is the Bush-Francis Catatonia Rating Scale BFCRS scale. This scale is versatile, readily available for clinic use, easy to use, and has been translated into many languages (Carroll et al., 2008).

\section{Diagnostic Conflict}

There exists strong syndromal overlap between NMS and malignant catatonia (Lang et al., 2014), so much so that the clinical distinction between the two diagnoses can be difficult to ascertain. In the DSM-IV, it was found that approximately $94 \%$ of patients meeting the criteria for NMS could also meet criteria for catatonia if using the BFCRS (Lang et al., 2014). Further muddying of the diagnostic waters stems from a lack of characteristic sequence to symptom presentation (Pileggi \& Cook, 2016). The DSM-5 has sought to better identify catatonia while also widening the definition of NMS (Loeb et al., 2015). This, combined with the 2014 review 
by Lang et al., has improved the chances of distinguishing the clinical symptoms more often associated with NMS (diaphoresis, rigor, fever, tremor, laboratory evidence of muscle injury, leukocytosis) from those of catatonia (negativism, posturing, waxy flexibility, stupor, and stereotypy). However, malignant catatonia remains clinically similar to NMS, with autonomic instability and fever. It is often only distinguishable when neuroleptics are not administered (Kumar et al., 2016).

\section{Treatment Algorithm}

The treatment for neuroleptic malignant syndrome begins with suspicion of the syndrome followed by discontinuation of the offending agents, usually antipsychotics that cause excessive dopamine blockade (Pileggi, Cook 2016). There have also been reports that the removal of prodopaminergic drugs, such as those used in Parkinson's disease, may induce NMS. In these cases, restarting the withdrawn medication is the most appropriate intervention (Pileggi \& Cook, 2016). Once NMS is suspected and the offending agent adjusted and supportive care initiated, selection of a reversal agent is key. Medications commonly used in treatment of NMS include dantrolene and, bromocriptine. Amantadine has been used as an adjunctive treatment in moderate to severe cases and benzodiazepines have commonly been used for additional symptomatic management (Pileggi \& Cook, 2016). When medications fail, electroconvulsive therapy has been found effective (Pileggi \& Cook, 2016).

Benzodiazepines, amobarbital, and electroconvulsive therapy have been effective treatments for catatonia (Carroll et al., 2008). Given the difficulty of distinguishing between the two conditions on initial presentation, a reasonable course is administration of Ativan followed by dantrolene if symptoms show no signs of improvement (Spiegel et al., 2019).

\section{Restarting Antipsychotic}

Restarting an antipsychotic after the occurrence of NMS should be done with great care, as rates of relapse can be as high as $30 \%$ (Rooijen, et al., 2017). Studies advocate a wash out period of 2 weeks or more before the reintroduction of antipsychotics. Furthermore, choosing a low potency antipsychotic and starting at a low dose with careful titration is recommended to help reduce the risk of relapse. In patients at high risk without medication, a shorter wash out period of 5 days can be tried, but only if the potential benefits outweigh the risks and careful monitoring is possible (Pileggi \& Cook 2016).

\section{Case Report:}

A married, 28 year old active duty male presented to an emergency room with a first break psychosis. He had an approximately one year history of major depressive disorder treatment that included successful trials of fluoxetine and bupropion XL. His past medical history was significant for alcohol counseling, although he did not meet criteria for an alcohol use disorder. Upon presentation, his wife reported that the patient had auditory and visual hallucinations, with response to internal stimulus. She also reported that he had experienced episodes of talking to an ex-girlfriend and his in-laws-who were not present. In response to these hallucinations, the patient began scratching his eyes vigorously. He claimed that he had been without sleep for about one week and, earlier on the day of presentation, had reported seizure-like activity to his wife. While in the ER, the patient became combative and was orally administered ziprasidone $20 \mathrm{mg}$, quetiapine $50 \mathrm{mg}$, and Ativan $1 \mathrm{mg}$, after which he was transferred to another hospital.

On arrival to the MTF the patient displayed paranoid delusions and continued to be combative. His presentation warranted chemical and physical restraints on the first day of hospitalization. Over the subsequent 3 days, to control his agitation and intermittent aggression toward staff, he received risperidone ( 12 doses of $1 \mathrm{mg}$ and one additional dose of $2 \mathrm{mg}$ ), haloperidone ( 3 doses of $5 \mathrm{mg}$ ), Ativan (3 doses of $2 \mathrm{mg}$ and 6 doses of $1 \mathrm{mg}$ ) and Cogentin ( 5 doses of $1 \mathrm{mg}$ ) in a combination of as needed and scheduled dosage (see Table 1k. Days in hospital and medication course). On the fourth day, the patient continued to have paranoid delusions and was observed to have racing thoughts, response to internal stimuli; low amplitude tremor with posture and action, though not at rest; restlessness; and stereotyped movements that later progressed to cataplexy and mutism. On day 4 the patient was thought to be in crisis. He exhibited autonomic instability, cataplexy, mild bilateral upper extremity tremor, and mutism. He also became unresponsive to a sternal rub. During this episode, it was thought the patient was having a seizure rather than a catatonic reaction and an immediate rapid response team (IRRT) was activated. His Bush-Francis Catatonia Rating Scale (BFCRS) score was elevated and noted for staring, catalepsy, rigidity, negativism, and withdrawal. He responded well to one dose of Ativan $2 \mathrm{mg}$ orally within minutes with a waning BFCRS score.

However, on day 6, another IRRT was activated for return of automatic instability, mutism, rigidity vs. negativism, and cataplexy as well as low grade fever (100.8). At this point he was transferred to the step down unit for concern for a malignant catatonia and possible 
NMS with recurring elevation of his BFCRS score. Laboratory testing showed an elevated creatine kinase (CK) 465 units/liters with slightly depressed white blood cell count (WBC) $4.2 \times 10^{3} / \mathrm{uL}$. The patient was given a single dose of dantrolene $85 \mathrm{mg}$ by mouth and all antipsychotics were stopped, which resulted in the complete resolution of symptoms (see Graph 1).

After a brief monitoring period the patient was transferred back to the psychiatric ward. He continued to receive scheduled Ativan and all antipsychotics were held with no recurrence of psychomotor agitation, NMS or catatonia. However, the patient continued to hallucinate throughout his hospital stay. His hallucinations at this point were in full consciousness. He was able to abate with acknowledgement and reality testing was intact. Vitals and laboratory work-up trended toward normal. Ultimately, the patient, on divalproex delayed release $500 \mathrm{mg}$ by mouth twice daily, was discharged with well controlled visual hallucinations, resolved autonomic dysregulation, full insight, good judgment and restoration of normal faculties without psychomotor agitation. By day 14, discharge diagnoses included Bipolar I Disorder, more recent episode manic with psychotic features and resolved catatonia related to Bipolar I Disorder.

Days in hospital and correlating BFCRS scores are graphed. Notice a peak in BFCRS scores after continuous administration of second generation antipsychotic up until the fourth day of admission. BFCRS started to decline after given dantrolene and higher dosages of Ativan.

\section{Discussion:}

In this case, the patient's presentation advanced over several days post treatment with three different second-generation antipsychotics for a first-time bipolar type I manic episode and within hours once the possibility of NMS or malignant catatonia was recognized. At that time, his symptoms insidiously progressed from apparent catatonia to what resembled NMS or malignant catatonia. The patient demonstrated stupor, mutism, diminished responses to voice and painful stimuli, stereotypic non-goal-directed behaviors, such as picking things up from the floor repetitively, responding to internal stimuli, and fixed gaze with occasional scanning of the room-all suggestive of catatonia (APA, DSM-5, 2013). Due to profound negativism and resistance to movement or inspection (lead pipe rigidity), muscle rigidity was appreciated. When muscle rigidity and low-grade fever were seen in the setting of autonomic instability, NMS became a concern.

At this time, the patient presented with agitation that evolved into profound mental status changes and finally into a state of stupor. He demonstrated superimposed bilateral hand tremor, low grade fever of 100.4 to $100.8 \mathrm{~F}$, and dysregulation of his vital signs with no dysrhythmias. Hours into this abrupt presentation, the patient developed elevation in creatine kinase, four times the upper limit of normal, with concern for rhabdomyolysis. However, he did not display sialorrhea, dysarthria, or dysphagia. A trial of Ativan to differentiate malignant catatonia from NMS did not interrupt the escalation of patient's symptoms (Pileggi \& Cook, 2016). Thus, at this point more of a concern for NMS than malignant catatonia was in question.

Eventually, the patient was transferred to the step-down unit (SDU) and stabilized. At the SDU, he was provided with dantrolene and antipsychotics were withdrawn. This resulted in good control of his heterogeneous symptoms. In spite of his receiving daily dosages of Ativan, his clinical picture progressed to a more neuroleptic malignant etiology when the antipsychotic was continued. This is suggestive of NMS, and ultimately, he responded to one dose of dantrolene combined with discontinuation of risperidone and higher dosages of Ativan. Prior to this point, the patient's dosage of Ativan was not upregulated and only reached a minimum range recommended for catatonia before his symptoms resolved. The overlap between the timing of the first dose of dantrolene, not receiving a robust amount of Ativan, and removal of risperidone make the diagnosis unclear. Another point to consider is that cessation of anti-psychotics is indicated as a treatment for NMS but not for catatonia. Considering all the factors presented, dantrolene could have been curative — or have had no impact at all; we cannot be certain.

\section{Pathophysiology}

There is agreement that NMS and malignant catatonia can be indistinguishable from each other. Specifically, catatonia with pyrexia and autonomic instability are blurred by pathophysiology. It has been stated that NMS is a subcortical motor syndrome caused by dopaminergic dysregulation, whereas malignant catatonia is a GABAergic dysregulation from the cortical psychomotor region of the brain (Reilly, et al., 2017).

The pathophysiology of NMS is thought to be related to a dopamine antagonist effect. This effect causes inhibition of dopamine availability thus heightening a lead pipe muscle rigid appearance and ineffective ability to regulate autonomic organ functions. 
Usually, NMS is a rare life-threatening reaction to antipsychotics that commonly presents with pyrexia, autonomic instability, rigidity, and altered consciousness (Reilly, et al., 2017). Eventually, this can lead to complications such as metabolic acidosis,

hypermetabolism, chest wall restriction, aspiration pneumonia or pulmonary emboli, and sudden respiratory arrest (APA, DSM-5, 2013).

The development course of NMS usually starts with altered mental status and varies from an onset of hours to days. Eventually, after the antipsychotic drugs are discontinued, resolution of symptoms resolves within one week. In our case, symptoms resolved within hours to one day of administration of multiple interventions, but most notably when the patient was given dantrolene, higher dosages of Ativan, withdrawal of antipsychotics and immediate intensive care was implemented. Notably, fatal rates have been reported to be between $10-20 \%$ when the diagnosis is unrecognized. Although most do not have a recurrence of NMS, some patients have a recurrence if antipsychotics are added back soon after the episode (APA, DSM-5, 2013). In our case antipsychotics were not attempted until one month later with no reoccurrence of NMS.

\section{Suggested Course of Action}

Our proposal is to have a high degree of suspicion when using antipsychotics per application of catatonia scales. Also, a trial of Ativan before administration of a more complex medication is warranted. Another topic considered in the literature is treatment of catatonia with therapeutic doses of Ativan (ranging from 8-24mg/day). Dual treatment with an antipsychotic and Ativan is another consideration (Spiegel et al., 2019). In our patient, it could be proposed that therapeutic dosage levels to prevent NMS and to treat catatonia were not reached given that a response was achieved after dantrolene was administered. It could also be suggested that either he was not given enough Ativan to prevent NMS, the dose of Ativan was not within therapeutic dosage ranges to treat catatonia, or he was given enough antipsychotic medication to induce NMS - or the diagnosis of NMS and malignant catatonia occurred concurrently.

The literature has suggested the need for more catatonia rating scales, specifically those that predict a surge in catatonia. Our patient's scores were slowly increasing with an insidious onset of catatonia, which we believe slipped into an NMS reaction. BFCRS rating scales are elevated when the patient clinically improves. This was demonstrated in our case and the patient continued to have an elevated score, even after the clinical concern for catatonia was not appreciated.

In a study by Kirkhart et al. (2007), some subjects continued to have a score between 3 -12 even after physiological stabilization was achieved. They found that the verbiage describing symptoms of catatonia was ambiguous and had poor validity. More accurate rating scales would be helpful in predicting or ruling out NMS (Kirkhart et al., 2007). In fact, Carroll et al. found that there is not enough sensitivity to determine the appropriate treatment response when using BFCRS scoring (2008). Scales that differentiate between malignant catatonia and NMS would be ideal.

\section{Limitations}

Clarity of diagnosis and validity of catatonia score scales to identify psychomotor syndromes are limited. Our case is anecdotal, and as a single case report, it is inherently limited. It is also understood that there is a blurring of the malignant catatonia and NMS diagnoses. One method suggested to distinguish between the two diagnoses is the use of neuroleptics implicating NMS versus a disease process such as malignant catatonia. For instance, catatonia is known to be a clinical consequence of various psychiatric DSM-5 diagnosis spectrums such as autism, schizophrenia, mood disorders and medical conditions. This has been supported by Asztalos (2014), who notes that there has been overlap in the neuropathological and pathophysiological aspects of the two diagnoses since the introduction of neuroleptics to treat catatonia began in the 19th century.

We may not have used the effective dosages of Ativan at the inception of his catatonic presentation and the level of similarity neuropathological and pathophysiological aspects of both diagnoses was not strongly considered. Catatonia dosage ranges were not achieved until the BFCRS scores were suspicious for prodromal NMS. At this point, the patient remained on a more therapeutic dose of Ativan for one day with worsening BFCRS scores. Not until dantrolene was utilized did the patient's clinical picture start to resolve. It could have been that his psychomotor syndrome was already starting to respond to more therapeutic dosages of Ativan or that he truly was exhibiting the beginning stages of NMS and it resolved with the initiation of dantrolene and cessation of antipsychotics.

However, we were relying on the clinical presentation and BFCRS scores, which are known to be subjective. Thus, BFCRS scales have variable sensitivity and the definitions used to measure resolution of psychomotor syndrome lack clarity. Although in this case, scores correlated with our patient's worsening clinical picture. Even after recovering clinically, his scores remained elevated, suggesting continuation of psychomotor syndrome. Additionally, his Ativan dosage was well below therapeutic levels during this time of elevated 
BFCRS score but he still continued to improve. Because all three were treated for at the same time, whether the patient had NMS, malignant catatonia, or catatonia syndrome remains unclear.

\section{Conclusion:}

Although our intervention resulted in a resolution of psychomotor presentation, the diagnosis, which was unclear initially, continues to be unclear, exemplifying the need for more precise scoring scales to differentiate between malignant catatonia, catatonia and NMS. Treatment was also a point of contention. If using Ativan to address catatonia, we recommend the use of published therapeutic ranges. In our case, therapeutic ranges could have prevented malignant catatonia. It is important to note that NMS can complicate psychiatric disorder if antipsychotic medication is given. Early suspicion of NMS and the treatment of both NMS and malignant catatonia probably saved our patient's life. However, Ativan dosage trials would be of great benefit. More prospective randomized studies should be initiated to establish diagnostic clarity. Finally, better psychomotor score scales to detect, monitor and determine severity should be developed.

\section{Declarations:}

No funding was required or received for this study

The authors have no conflicts of interest

NMRTC IRB/ethics approval is not required for case reports

Consent for treatment and publication of case was obtained

No data was gathered on this single patient

Author Contributions:

Dynela Garcia-Baran: Conception and design of the work, executed literature search and final revision of the article.

Sam Collier: Conception and design of the work, and executed literature search.

Alejandro Ortiz: Critical execution of revisión and literature search of the article.

\section{References:}

1. American Psychiatric Association. (2013). Diagnostic and Statistical Manual of Mental Disorders, Fifth Edition, Text Revision. Washington, DC. Section 2 - Diagnostic codes on medication-induced movement disorders and other adverse of medication. Page 70.

2. Asztalos Z, Egervari L, Andrassy G, Faludi G, Frecska E. (2014). Catatonia and neuroleptic malignant syndrome in view of a psychopathological and pathophysiological overlap: a brief review. Neuropsychopharmacology Hungary, Mar 16(1), 19-28.

3. Carroll BT, Kirkhart R, Ahuja N, Soovere I, Lauterbach EC, Dhossche D, \& Talbert R. (2008). Katatonia: a new conceptual understanding of catatonia and a new rating scale. Psychiatry (1550-5952), 5(12), 42-50. Retrieved from http://search.ebscohost.com/login.aspx?direct=true\&db=rzh\&AN=105611583\&site=ehost-live

4. Kirkhart R, Ahuja N, Lee JW, Ramirez J, Talbert R, Faiz K, Ngvari GS, Thomas C, Carroll BT. (2007). The Detection and Measurement of Catatonia. Psychiatry (Edgmont), Sep 4(9), 52-56.

5. Kumar KK, Bondade S, Sattar FA, and Singh N. (2016). Malignant Catatonia and Neuroleptic Malignant Syndrome in Relation to Disulfiram Overdose. Indiana Journal Psychology Medicine, Jul-Aug 38(4), 344-347. doi: 10.4103/0253-7176.185944

6. Lang, Fabian \& Lang, Silke \& Becker, Thomas \& Jäger, Markus. (2014). Neuroleptic malignant syndrome or catatonia? Trying to solve the catatonic dilemma. Psychopharmacology. 232. 10.1007/s00213-014-3807-8.

7. Loeb, E., Madigand, J., Alexandre, J., Dollfus, S., Coquerel, A., \& Fedrizzi, S. (2015). Neuroleptic malignant syndrome and catatonia overlapping: 2 case reports. Psychopharmacology, 232(14), 2643-2644. doi: 10.1007/s00213-015-3985-z 
8. Pileggi, D. J., \& Cook, A. M. (2016). Neuroleptic Malignant Syndrome: Focus on Treatment and Rechallenge. Annals of Pharmacotherapy, 50(11), 973-981. doi: 10.1177/1060028016657553

9. Reilly, T. J., Cross, S., Taylor, D.M., Haslam, R., Tomlin, S.C. \& Gaastra, B. (2017). Neuroleptic malignant syndrome following catatonia: Vigilance is the price of antipsychotic prescription. SAGE Open Med Case Rep, 5, 1-6.

http://dx.doi.org/10.1177/2050313X17695999.

10. Spiegel DR, Glad R, Smith M, Raja U, Wade R, Johnson K. (2019). A Case of Schizophrenia With Catatonia Resistant to Ativan and Olanzapine Monotherapy But Responsive to Combination Treatment: Is It Time to Consider Using Select Second-Generation Antipsychotics Earlier in the Treatment Algorithm for This Patient Type? Clinical Neuropharmacology, 42(2), 57-59.

\section{Table:}

Table 1

Days in hospital and medication course

\begin{tabular}{|c|c|c|c|c|c|c|c|c|c|c|}
\hline LOS & $\begin{array}{l}\text { Risperidone } \\
\text { Dosage }\end{array}$ & $\begin{array}{l}\text { Divalproex } \\
\text { Dosage }\end{array}$ & $\begin{array}{l}\text { Quetiapine } \\
\text { Dosage }\end{array}$ & $\begin{array}{l}\text { Ziprasidone } \\
\text { Dosage }\end{array}$ & $\begin{array}{l}\text { Haloperidone } \\
\text { Dosage }\end{array}$ & $\begin{array}{l}\text { Ativan } \\
\text { Dosage }\end{array}$ & $\begin{array}{l}\text { Dantrolene } \\
\text { Dosage }\end{array}$ & CK & WBC & BFCRS \\
\hline 1 & $1 \mathrm{mg}$ bid & & & $50 \mathrm{mg} \times 1$ & $5 \mathrm{mg}$ & $5 \mathrm{mg}$ & $(-)$ & & & 9 \\
\hline 2 & $2 \mathrm{mg}$ bid & & & & & $2 \mathrm{mg}$ & & & & 9 \\
\hline 3 & $2 \mathrm{mg}$ bid & & & & & 3 & & & & 15 \\
\hline 4 & $\begin{array}{l}3 \mathrm{mg} \mathrm{bid} \\
\text { discont'd }\end{array}$ & & & & & 1 & & & & 19 \\
\hline 5 & & & $50 \mathrm{mg} \times 1$ & $50 \mathrm{mg} \times 1$ & & 9.5 & & 306 & 3.2 & 19 \\
\hline 6 & & $\begin{array}{l}500 \mathrm{mg} \\
\mathrm{pm}\end{array}$ & & & & 8.5 & $85 \times 1$ & 465 & 4.2 & 21 \\
\hline 7 & & $\begin{array}{l}500 \mathrm{mg} \\
\text { bid }\end{array}$ & & & & 7 & & 200 & 4.2 & 11 \\
\hline 8 & & $\begin{array}{l}500 \mathrm{mg} \\
\text { bid }\end{array}$ & & & & 3 & & & & 7 \\
\hline 9 & & $\begin{array}{l}500 \mathrm{mg} \\
\text { bid }\end{array}$ & & & & 3 & & & & 2 \\
\hline 10 & & $\begin{array}{l}500 \mathrm{mg} \\
\text { bid }\end{array}$ & & & & 3 & & & & 0 \\
\hline 11 & & $\begin{array}{l}500 \mathrm{mg} \\
\text { bid }\end{array}$ & & & & 5 & & & & 0 \\
\hline 12 & & $\begin{array}{l}500 \mathrm{mg} \\
\text { bid }\end{array}$ & & & & 2 & & & 3.6 & 0 \\
\hline 13 & & $\begin{array}{l}500 \mathrm{mg} \\
\text { bid }\end{array}$ & & & & 1.5 & & & & 0 \\
\hline 14 & & $\begin{array}{l}500 \mathrm{mg} \\
\text { bid }\end{array}$ & & & & 0.5 & & & & 0 \\
\hline
\end{tabular}

BFCRS $=$ Bush-Francis Catatonia Rating Scale, LOS = Length of Stay and CK = creatine kinase.

\section{Figures}




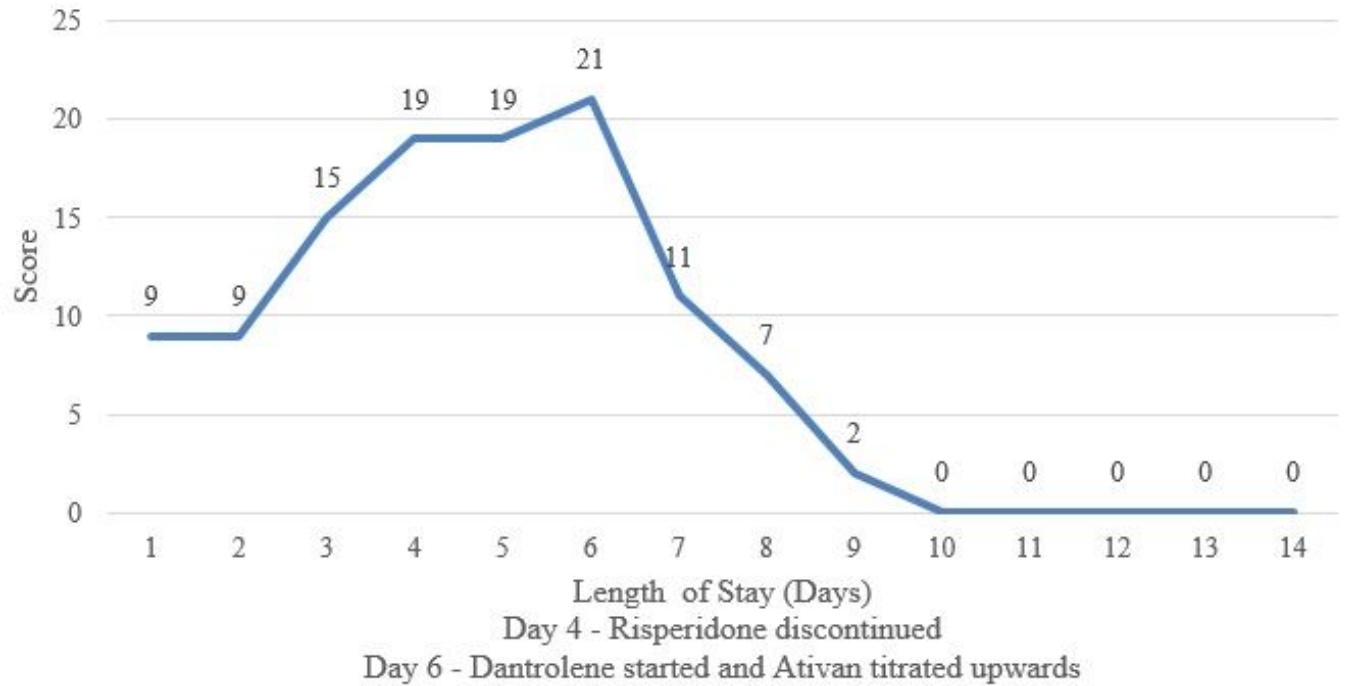

\section{Figure 1}

BF Catatonia Rating Scale \& Medication.

\section{Supplementary Files}

This is a list of supplementary files associated with this preprint. Click to download.

- CAREchecklistEnglish2021NMSv.maligcatatonia.pdf 